\title{
RNA G-quadruplex structures exist and function in vivo
}

Xiaofei Yang ${ }^{1}$, Jitender Cheema ${ }^{1}$, Yueying Zhang ${ }^{1}$, Hongjing Deng ${ }^{1,2,3}$, Susan Duncan ${ }^{1}$, Mubarak Ishaq Umar ${ }^{4}$, Jieyu Zhao ${ }^{4}$, Qi Liu ${ }^{1,5}$, Xiaofeng Cao ${ }^{2,3,{ }^{*}}$, Chun Kit Kwok ${ }^{4, *}$, Yiliang Ding ${ }^{1, *}$

1.Department of Cell and Developmental Biology, John Innes Centre, Norwich Research Park, Norwich NR4 7UH, United Kingdom

2. College of Life Sciences, University of Chinese Academy of Sciences, 100049, Beijing, China

3. State Key Laboratory of Plant Genomics and National Center for Plant Gene Research, Institute of Genetics and Developmental Biology, Chinese Academy of Sciences. Beijing 100101, China

4. Department of Chemistry, City University of Hong Kong, Kowloon Tong, Hong Kong SAR, China

5. Present address: School of Life Sciences, University of Sussex, Brighton, BN1 9QG, UK

*Correspondence: yiliang.ding@jic.ac.uk, ckkwok42@cityu.edu.hk and xfcao@genetics.ac.cn 


\section{Abstract}

Guanine-rich sequences are able to form complex RNA structures termed RNA G-quadruplexes in vitro. Because of their high stability, RNA G-quadruplexes are proposed to exist in vivo and are suggested to be associated with important biological relevance. However, there is a lack of direct evidence for RNA G-quadruplex formation in living cells. Therefore, it is unclear whether any purported functions are associated with the specific sequence content or the formation of an RNA G-quadruplex structure. Here, we profiled the landscape of those guanine-rich regions with the in vitro folding potential in the Arabidopsis transcriptome. We found a global enrichment of RNA G-quadruplexes with two G-quartets whereby the folding potential is strongly influenced by RNA secondary structures. Using in vitro and in vivo RNA chemical structure profiling, we determined that hundreds of RNA G-quadruplex structures are strongly folded in both Arabidopsis and rice, providing direct evidence of RNA G-quadruplex formation in living eukaryotic cells. Subsequent genetic and biochemical analysis showed that RNA G-quadruplex folding was sufficient to regulate translation and modulate plant growth. Our study reveals the existence of RNA Gquadruplex in vivo, and indicates that RNA G-quadruplex structures act as important regulators of plant development and growth. 


\section{Introduction}

The in vivo folding of RNA structure is tightly associated with its function and largely dependent on cellular context ${ }^{1,2}$. Because of the complexity within living cells, RNA folding in vivo could be very different from that in vitro ${ }^{3,4}$. For many complex RNA structures, although their folding in vitro has been well defined, the folding in vivo is poorly understood. One of such complex structures is RNA G-quadruplex (RG4), which is folded with guanine-rich (G-rich) sequences in vitro and consists of two or more layers of G-quartets involving both Hoogsteen and Watson-Crick base pairs ${ }^{5,6}$. RG4s can be very stable in vitro in the presence of ion cations such as potassium $\left(\mathrm{K}^{+}\right)$, therefore they are hypothesized to exist in vivo and to be involved in novel functions $^{5}$, such as post-transcriptional regulation of gene expression ${ }^{7-9}$. Nevertheless, the lack of direct evidences of RG4 folding in vivo raised the key question of whether all these suggested functions are due to RG4 structure or sequence motif. For example, a (GGC) 4 motif was proposed to fold into an RG4 structure to repress translation of tumor-related genes ${ }^{8}$. However, without the evidence of in vivo folding, the translation inhibition may simply be due to the (GGC) 4 sequence motif. Also, emerging evidences argues that this sequence motif is likely to form a stable hairpin RNA secondary structure rather than $\mathrm{RG} 4^{10}$. Hence, it is crucial to determine whether RG4 truly exists in vivo, such that one can investigate and establish the relationship between RG4 and associated biological functions.

In recent decades, numerous efforts have been made to detect the folding of RG4s in fixed or living cells using G-quadruplex-specific antibodies ${ }^{11}$, ligands ${ }^{12-14}$ and fluorescent probes ${ }^{15,16}$. However, these methods suffer from three major shortcomings. Firstly, the antibodies / ligands / probes may induce the structure formation by perturbing the RNA G-quadruplex folding equilibrium in cells or binding to the G-rich sequence motifs ${ }^{17,18}$. Secondly, these methods cannot 
quantitively determine the folding state of individual G-rich regions of interest in cells. Thirdly, these methods cannot exclude the possibility of RG4 folding in fixing, permeabilizing or staining cells ${ }^{17}$. Because of these considerable shortcomings, these methods are considered inadequate for robustly determining the existence of RG4s and actual folding state of G-rich regions in living cells.

To address these shortcomings, two methods based on RNA chemical structure probing have been developed to determine folding state of G-rich regions in living cells. One direct method is based on the property of the chemical 2-methylnicotinic acid imidazolide (NAI) which preferentially modifies the last Gs of G-tracts when RG4 is folded. This special modification pattern is subsequently detectable by reverse transcription at both individual targeted RNAs and at a genome-wide scale ${ }^{17,19}$. The other method is more indirect, the modification is based on the specific methylation of the $\mathrm{N} 7$ position of guanine $(\mathrm{N} 7 \mathrm{G})$ by dimethyl sulfate (DMS) ${ }^{20}$. When a very high concentration of DMS $(\sim 8 \%)$ is applied to the cells, all the N7 positions of G residues in the unfolded G-rich regions will be methylated in vivo ${ }^{17}$. These methylated G-rich regions are unable to refold into RG4 structure in vitro in the presence of $\mathrm{K}^{+}$. Since $\mathrm{RG} 4$ refolding in vitro are able to stall reverse transcriptase during reverse transcription $(\mathrm{RT})^{21}$, in vivo unfolded G-rich regions are unable to lead to RT stalling ${ }^{17}$. On the opposite, if the G-rich regions are folded into RG4 structures in vivo, then N7G is protected from DMS modification. These unmodified G-rich regions are able to reform into RG4 structures later during $\mathrm{RT}$, subsequently causing the RT stalling ${ }^{17}$. Both methods were applied in yeast and the DMS method was also applied in mouse embryotic stem cells (mESCs) ${ }^{17}$. These studies concluded that G-rich regions with the potential to form RG4s in vitro were globally unfolded in vivo. These results underpinned the fact that both 
yeast and mice avoid the formation of RG4s in vivo, and the presumption that RG4s do not exist in eukaryotic cells ${ }^{17}$.

Unlike yeast and animals, plants are sessile eukaryotic organisms and have evolved independently with unique regulatory strategies. For instance, cellular $\mathrm{K}^{+}$is the most abundant ion in plants and plays key roles in plant development and stress response $\mathrm{e}^{22,23}$. Given the importance of $\mathrm{K}^{+}$in affecting RG4 folding, and the ability of plants in maintaining $\mathrm{K}^{+}$balance within the cells, we hypothesize that plants are more likely to adopt RG4 structure in vivo. In addition, our previous study and others on individual G-rich sequences with folding potential in vitro have suggested that 85 plants might favor RG4 structures ${ }^{9,24}$. However, the existence of RG4s in vivo in plants has not been determined and remains an open question.

Here, we investigated the in vivo folding state of G-rich regions in plants. Firstly, we profiled the landscape of the G-rich regions with the potential to fold into RG4s in vitro in the Arabidopsis transcriptome. We also revealed the unique RNA structural features of these regions. Using 90 chemical structure probing, we found that those G-rich regions with in vitro folding potential are strongly folded in both Arabidopsis and rice. We further demonstrated that RG4 formation is sufficient to regulate gene expression, and subsequently plant growth. Taken together, these findings provided the first direct evidence of global RG4 formation in living eukaryotic cells, and revealed RG4s as important regulators for plant growth. 


\section{Results}

\section{Profiling of G-rich regions with potential to fold into RG4 structures in Arabidopsis transcriptome}

To systematically search for G-rich regions with the potential to fold into RG4 structures in Arabidopsis, we used rG4-seq, an in vitro approach for detecting RG4 structures formation at a transcriptome-wide scale ${ }^{25}$. RG4 formation in vitro is stabilized by potassium ions ( $\mathrm{K}^{+}$) (Fig. 1a), but not lithium ions $\left(\mathrm{Li}^{+}\right)$, and is preferentially stabilized by G-quadruplex stabilizing ligands such as pyridostatin $(\mathrm{PDS})^{26}$. G-rich regions which folded into RG4 structures in vitro can cause reverse transcriptional stalling (RTS) ${ }^{21,25}$. Therefore, RTS sites dependent on the presence of $\mathrm{K}^{+}$or $\mathrm{K}^{+}+\mathrm{PDS}$ suggest the presence of G-rich regions with RG4 folding potential within sequences upstream of the RTS (Fig. 1b). We performed reverse transcription on Arabidopsis RNAs with $\mathrm{Li}^{+}, \mathrm{K}^{+}$or $\mathrm{K}^{+}+\mathrm{PDS}$ respectively and generated corresponding libraries with high reproducibility (Supplementary information Fig. S1). To validate our rG4-seq, we mapped RT stops on the mRNA of SUPPRESSOR OF MAX2 1-LIKE5 (SMXL5) which contains a G-rich region with RG4 folding potential identified recently9. We found a strong RT stalling at the 3'end of this G-rich region where the coverage dropped in the presence of both $\mathrm{K}^{+}$and $\mathrm{K}^{+}+\mathrm{PDS}$ conditions, but not $\mathrm{Li}^{+}$(Fig. 1c), agreeing with the previous gel-based assay ${ }^{9}$.

We then searched for RTS sites dependent on the presence of $\mathrm{K}^{+}$or $\mathrm{K}^{+}+\mathrm{PDS}$ in the Arabidopsis transcriptome. Our meta-analysis showed that guanine (G) was strongly enriched in sequences upstream but not downstream of RTS sites, for both $\mathrm{K}^{+}$and $\mathrm{K}^{+}+\mathrm{PDS}$ conditions (Fig. 1d). A strong enrichment of guanine suggested the prevalence of G-rich regions with potential to form RG4 structures in vitro ${ }^{17,25}$. We searched for G-rich regions able to form RG4 structures on the basis of special sequence feature, GxLnGxLnGxLnGx (whereby G stands for Guanine; L 
stands for Loop, and $\mathrm{x} \geq 2$, $\mathrm{n}$ up to 15 , see methods) in sequences upstream of RTS. In total, we (Supplementary information Table S1, Table S2). We then classified these G-rich regions according to G-quartet number, loop length and bulge size. In the presence of $\mathrm{K}^{+}$, we found 253 G2 (with two G-quartets) and 128 G3 (with three G-quartets) G-rich regions (Fig. 1e and Supplementary information, Table S1). In the presence of $\mathrm{K}^{+}+\mathrm{PDS}$, we detected $2234 \mathrm{G} 2$ and 223 G3 regions (Fig. 1e and Supplementary information, Table S2). As illustrated by rG4-seq profiles of individual regions on $A T 4 G 30460$ and $A T 3 G 23450$, coverage dropped down in the presence of $\mathrm{K}^{+}$and $\mathrm{K}^{+}+\mathrm{PDS}$, but not $\mathrm{Li}^{+}$, at the 3'end of these G-rich regions, indicating folding of G2 and G3 RG4 structures (Fig. 1g, h). We also examined the location of these G-rich regions, and found that they were mostly localized in the coding regions (Fig. 1f, Supplementary information, Table S1 and Table S2). Taken together, we revealed the global in vitro landscape of G-rich regions with the potential to fold into RG4 structures in the Arabidopsis transcriptome.

\section{Features of Arabidopsis RG4 formable regions}

Over 65,000 G-rich regions were predicted in silico to form RG4 structures in Arabidopsis

135 based on the sequence feature of GxLnGxLnGxLnGx ${ }^{6,27}$. However, we detected less than 3000 regions by rG4-seq (Fig. 1e and Supplementary information, Table S1, Table S2), indicating most of predicted regions are unlikely to fold into RG4 structures in vitro. This prompted us to ask whether this low rate of in vitro folding is due to competition from alternative secondary structure formation, which might prevent RG4 formation across these predicted regions. To test this 140 hypothesis, we used Vienna RNAfold software to predict the secondary structures of both in silico predicted but undetected G-rich regions and the detected RG4 regions by rG4-seq with $\mathrm{K}^{+28}$. We 
145 flanking regions (Fig. 1i, $\mathrm{P}<10^{-16}$, paired Student's t-test), indicating these G-rich regions were folded into strong secondary structures. In contrast, for those detected G-rich regions in the presence of $\mathrm{K}^{+}$, no significant differences of BPPs were found between G-rich regions and flanking sequences (Fig. 1i, P = 0.444, paired Student's t-test), and the BPPs of G-rich regions are strongly lower than that of the undetected regions (Fig. 1i, $\mathrm{P}<10^{-16}$, Student's t-test). Therefore, those 150 regions detected in vitro by rG4-seq are likely to form weak secondary structure while the undetected regions are likely to fold into strong secondary structures (Fig. 1j and Supplementary information, Fig. S2). Taken together, our results indicate that alternative secondary structures in those G-rich regions strongly affect the potential of folding into RG4 structures in vitro.

\section{SHALiPE-Seq robustly determines the folding state of G-rich regions}

Next, we asked if these G-rich regions with folding potential in vitro are able to fold into RG4 structures in vivo. Our previous studies have shown that both NAI and DMS are capable of penetrating plant cells ${ }^{29,30}$. We selected NAI rather than DMS for our in vivo determination of the folding state of the G-rich regions in plants, because the DMS method using high DMS unpredictable inaccuracies of structure determination implied by the high concentration of DMS method, we employed the NAI chemical probing method by coupling our previous method selective 2'-hydroxyl acylation with lithium ion-based primer extension (SHALiPE) with high throughput sequencing, which we termed SHALiPE-Seq ${ }^{19,29}$. 
SHALiPE-Seq is based on the preferential modification of the last $G$ in $G$ tracts of folded RG4s by 2-methylnicotinic acid imidazolide (NAI) (Fig.2a) ${ }^{17,19,32}$. Strong modifications are able to cause reverse transcription stalling on these last guanines, which are detectable by deep sequencing. Significantly higher reads number can be found on these last guanines for folded Grich regions, rather than unfolded ones (Fig. 2a). This method could discriminate the folded state from unfolded state of individual G-rich regions by showing reads number with or without the special pattern. Before applying this method to plants in vivo, we firstly established benchmark SHALiPE profiles for both folded and unfolded states of G-rich regions in plants in vitro in the presence of $\mathrm{K}^{+}$(folded state) or $\mathrm{Li}^{+}$(unfolded state), respectively (Fig. 2a, see methods). We assured our NAI modification efficiencies in the presence of $\mathrm{K}^{+}$and $\mathrm{Li}^{+}$were similar, as indicated 175 by gel-based analysis on $18 \mathrm{~S}$ rRNA (Supplementary information, Fig. S3a). We then generated the corresponding SHALiPE-Seq libraries with high reproducibility (Supplementary information, Fig. S3b, c).

In these SHALiPE-Seq libraries, the distribution of read counts on guanines for the folded state (with $\mathrm{K}^{+}$) is uneven, while the distribution for the unfolded state (with $\mathrm{Li}^{+}$) is uniform (Fig. $1802 \mathrm{a})^{17}$. These distributions were measured using the Gini index, where a high Gini indicates an uneven distribution (folded state) and a low Gini indicates a uniformed distribution (unfolded state) (Fig. 2a) ${ }^{17}$. As expected, for the G-rich regions detected by rG4-seq, the Gini of SHALiPE profiles in vitro with $\mathrm{K}^{+}$was greater than that in vitro with $\mathrm{Li}^{+}$by a factor of 1.20 (Fig. $2 \mathrm{~b}, P<10^{-9}$, paired Student's t-test and Supplementary information, Table S3, reads $\geq 50 / \mathrm{nt}$ ). As illustrated by the individual G2 and G3 regions on $A T 2 G 05380$ and $A T 5 G 62670$ identified by rG4-seq (Fig. 2c, d), high reads counts were found on the last Gs (indicated with black arrows) in the SHALiPE profiles with $\mathrm{K}^{+}$, indicating the preferential NAI modification on these Gs when RG4s are formed (Fig. 2e, 
f, top channels). Conversely, reads counts in the SHALiPE profile with $\mathrm{Li}^{+}$are more uniform, representing the unfolded state of these G-rich regions (Fig. 2e, f, bottom channels). As a result, the Ginis of the SHALiPE profiles in the presence of $\mathrm{K}^{+}(0.67$ and 0.52$)$ are higher compared to the corresponding 0.52 and 0.35 for $\mathrm{Li}^{+}$(Fig. 2e, f, text annotation on upper right). These results indicate that SHALiPE-Seq and rG4-seq are in strong mutual agreement, and that SHALiPE-Seq profiling is able to determine the folding state of individual G-rich regions at the transcriptomewide scale.

\section{Stable folding of RG4 structures in Arabidopsis in vivo}

To assess the in vivo folding state of these G-rich regions, we firstly performed in vivo NAI chemical probing in Arabidopsis ${ }^{29}$, and generated in vivo SHALiPE-Seq libraries with high reproducibility (Supplementary information, Fig. S3d). We then compared SHALiPE profiles in vivo with our benchmark SHALiPE profiles in vitro for both folded and unfolded states (Fig. 3a) ${ }^{17,19,29}$. Next, we calculated the Gini in vivo of G-rich regions and found that Gini in vivo was greater than that in vitro with $\mathrm{Li}^{+}$by a factor of 1.10 (Fig. 3b, $P<10^{-16}$, paired Student's t-test and Supplementary information, Table $\mathrm{S} 4$, reads $\geq 50 / \mathrm{nt})$. This result suggested that these G-rich regions are folded into RG4 structures in vivo. To further quantify the folding state in vivo, we calculated the in vivo folding score: a comparison of Gini in vivo with Gini in vitro in the presence of $\mathrm{Li}^{+}$, scaled relative to Gini in vitro with $\mathrm{K}^{+}$versus Gini in vitro with $\mathrm{Li}^{+17}$. In vivo folding scores of these G-rich regions centered near 1 with a median value of 0.755 (Fig. 3c and Supplementary information, Table S4), indicating that most G-rich regions identified by rG4-seq were strongly folded in Arabidopsis. The in vivo folding of RG4s in Arabidopsis differs from the unfolded states in yeast and mouse embryonic stem cells, where the folding scores centered near 0 (median values 
of -0.02 and 0.06 respectively $)^{17}$. The in vivo SHALiPE profile resembled the in vitro SHALiPE profile in the presence of $\mathrm{K}^{+}$(folded state), but not in the presence of $\mathrm{Li}^{+}$(unfolded state) for individual G-rich regions, as exemplified by regions on $A T 3 G 23450$ and $A T 4 G 30460$ (Fig. 3d, e). We further assessed whether any specific type of G-rich regions may be preferentially folded in vivo, but found very similar folding scores among different types of RG4s indicating no specific preference (Fig. 3f). In addition, the folding scores for RG4s in both coding regions (CDS) and untranslated regions (UTRs) were quite similar (Fig. 3g), indicating both CDS and UTRs contain stable RG4 structures in vivo. Taken together, our results indicate that in Arabidopsis, hundreds of G-rich regions are strongly folded into RNA G-quadruplexes in vivo, unlike the previously reported in vivo observations in yeast and mice ${ }^{17}$.

\section{In vivo folding of RG4 structures in rice}

To determine whether RG4s exist in diverse plant species, we investigated the folding state of G-rich regions in rice (Oryza sativa ssp. japonica), one of the most globally important $\operatorname{crops}^{33}$. We performed SHALiPE-Seq profiling in rice, and compared in vivo SHALiPE profiles with in vitro with $\mathrm{K}^{+}$and $\mathrm{Li}^{+}$respectively (Fig. 4a). Similar to observations in Arabidopsis, Gini in vivo for rice was greater than that in vitro with $\mathrm{Li}^{+}$by a factor of 1.20 (Fig. $4 \mathrm{~b}, P<10^{-16}$, paired Student's t-test and Supplementary information, Table S5). In vivo folding scores centered near 1 with a median value of 0.938 , indicating the folding status of RG4s in rice (Fig. 4c and Supplementary information, Table S5). The in vivo SHALiPE profile resembled the in vitro SHALiPE profile in the presence of $\mathrm{K}^{+}$(folded state) but not $\mathrm{Li}^{+}$(unfolded state) for individual $\mathrm{RG} 4 \mathrm{~s}$, as exemplified by regions on $L O C \_O s 02 g 15810$ and LOC_Os07g41694 (Fig. 4d, e). Moreover, no significant differences were found of the folding scores for RG4s of specific types nor different genic 
locations (Fig. 4f, g). Taken together, as represented by model dicot and monocot plant species, our results suggest the general existence of RG4s in the plant kingdom.

\section{RG4 structure regulates translation and plant development}

Considering our demonstration of the prevalence of RG4s in plants, and that RG4s are hypothesized to be associated with post-transcriptional regulation of gene expression ${ }^{5,6}$. We wanted to test the hypothesis by examining whether RG4s function in vivo. We focused on those in vivo folded RG4s (folding score $>0.5$ ) located in UTRs of genes in Arabidopsis (Supplementary information, Table S4). We screened T-DNA mutants of these genes and successfully identified homozygotes for gene HIRD11, which encodes a KS-type dehydrin and contains a G2 RG4 in its 3'UTR (Fig. 5a,b and Supplementary information, Fig. S4a). The mRNA abundance of HIRD11 in the mutant (termed hird11-1) was strongly reduced compared to wildtype Col-0 (Fig. 5c). The growth of hird11-1 was largely retarded relative to Col-0, represented by shorter primary roots compared to Col-0 (Fig. 5e, f). The phenotype of shorter primary roots in hird11-1 was restored by complementing mutants with HIRD11 containing wildtype RG4 sequence (wtRG4), indicating HIRD11 promotes plant growth (Fig. 5d, e, f). Strikingly, when hird11-1 mutant was complemented with HIRD11 containing mutated RG4 sequence (mutRG4, G to A mutation to disrupt RG4 folding capacity, Fig. 5d), the primary root length of mutRG4 plants was distinctively longer than that of wtRG4 plants (Fig. 5e, f).

We assessed whether RG4 folding affected the gene expression of HIRD11. Firstly, we compared the mRNA abundance of HIRD11 in wtRG4 and mutRG4 plants, and found no significant difference of mRNA between these plants (Supplementary information, Fig. S4b). We 
antibody against HIRD11 is available, we used polysome analysis by combining sucrose gradient fragmentation with qRT-PCR to detect the translational level of HIRD11 in these plants ${ }^{34,35}$. We found that polysome associated HIRD11 mRNA in mutRG4 plants was much higher than that in wtRG4 plants (Fig. 5g), indicating a higher translational level of HIRD11 in mutRG4 plants. Therefore, our results indicate the RG4 on HIRD11 suppresses its translation to modulate plant growth and development. 


\section{Discussion}

'If G-quadruplexes form so readily in vitro, nature will have found a way of using them in vivo' (Statement by Sir Aaron Klug over 30 years ago) ${ }^{36}$. Following decades of research by the community exploring RG4 structure across living eukaryotic cells, here for the first time, we determined hundreds of RG4s folded in model dicot and monocot plant species, providing direct evidence of RG4 existence in eukaryotes (Figs. 3 and 4). By both genetic and biochemical validation, we also demonstrated the important roles of the RG4 structure in modulating plant growth and development (Fig. 5).

RG4 structures contains unique sequence features of GxLnGxLnGxLnGx (whereby G stands for Guanine; L stands for Loop, and $\mathrm{x} \geq 2, \mathrm{n}$ up to 15 ). For decades, in silico prediction based on sequence features was used to search for putative RG4s at the genome-wide scale in different organisms $6,27,37,38$. With the rise of deep sequencing methods, high throughput methods such as rG4-seq were developed to map G-rich regions with in vitro RG4 folding potential throughout the transcriptome ${ }^{25}$. Here, we performed rG4-seq to map these G-rich regions in the Arabidopsis transcriptome. We identified less than 3000 G-rich regions with RG4 folding potential in vitro (Supplementary information Table S1, Table S2). In contrast, the in silico sequence-based method predicted over 65,000 G-rich regions ${ }^{27}$. Following our assessment of RNA secondary structures, we found that alternative secondary structures within those G-rich regions folded into might compete with RG4 structure formation (Fig. 1i and $\mathrm{j}$ ). If the G-rich region is able to form strong secondary structure, it is unlikely to fold into RG4 structure. This might be due to the slower folding kinetics for RG4 structure formation compared to the formation of alternative secondary structures $^{39}$. Thus, from a transcriptome-wide perspective, RG4 folding capability is generally 
influenced by alternative secondary structure formation, which may be an important way of regulating RG4 folding potential.

Following our rG4-seq in vitro profiling, we also revealed unique features for G-rich regions with RG4 folding potential in plants. We found that G-rich regions with potential to form G2 RG4s rather than G3 RG4s are predominate (>90\%) in plants (Fig. 1e, Supplementary information Table S1, Table S2), whereas in human cells, G-rich regions with potential to fold G3 RG4s are dominant ${ }^{25}$. Rather than the very stable structures that G3 RG4s fold ( $\left.\mathrm{Tm}>55^{\circ} \mathrm{C}\right), \mathrm{G} 2$ RG4s are less stable $\left(\mathrm{Tm} \sim 14-30{ }^{\circ} \mathrm{C}\right)^{40,41}$, thereby harbouring higher flexibility to switch between folded and unfolded states within the temperature range that most plants favour. This flexibility may facilitate a regulatory role in plant adaption to the immediate local environment. Rather than the depletion of G-regions for folding into RG4 structure in bacteria or avoiding formation of stable G3 RG4s in animals ${ }^{17}$, plants seem to have evolved a preference for G2 RG4 formation, which might be one of the reasons why plants have adopted RG4 structure as important regulators.

The large number of RG4s present in plants (Figs. 3 and 4) is probably explained by the physiological conditions in plants being favourable towards RG4 structure formation. For example, potassium $\left(\mathrm{K}^{+}\right)$is the predominant cytoplasmic inorganic cation in plants with a concentration around $100 \mathrm{mM}^{42,43}$, which is preferable for RG4 folding. Notably, unlike animals, plants lack a potassium/sodium exchanger and thus use a unique accumulation and release system for potassium ${ }^{43}$. Since RG4 folding is highly sensitive to potassium levels, RG4 structures have great potential to be adopted as a regulator in response to dynamic potassium level changes. Our gene ontology analysis (Supplementary information, Fig. S5) confirmed that genes involved in metal ion binding are significantly enriched (Fig. S5). As such, RG4 structures might contribute 
to maintaining an optimum potassium level in plants. Apart from potassium levels, temperature is another key factor that was suggested to affect the folding of RG4s in vitro ${ }^{40,41}$. The optimal environmental temperature for plants $\left(21-22{ }^{\circ} \mathrm{C}\right.$ for Arabidopsis and $26-28{ }^{\circ} \mathrm{C}$ for rice) is much lower than the body temperature of animals $\left(37^{\circ} \mathrm{C}\right.$ for human and $36.6{ }^{\circ} \mathrm{C}$ for mice). Thus, this relatively low temperature may allow the stable formation of RG4 structures in plant cells. In addition, a recent study found that the Arabidopsis RNA binding protein, zinc-finger protein JULG1 preferentially binds to a specific G-rich sequence with in vitro folding potential, stabilizing the RG4 structure in vitro even in the absence of potassium 9 . This result indicated that co-factors 315 such as RNA binding proteins might be important regulators for the formation of RG4 structures in plants. Notably, in vivo folding scores of many individual regions (60 out of 181 in Arabidopsis, $\sim 1 / 3$ ) are over 1 (Fig. 3c and Supplementary information, Table S4), indicating the in vivo folding state could be stronger than the in vitro folding state in the presence of $\mathrm{K}^{+}$. This result suggested that other factors might also promote RG4 formation in vivo. Hence, the ideal physiological conditions in plants together with those co-factors may confer plants with the ability to adopt RG4 structures, as regulators for gene expression.

Another interesting observation from our study is the variable folding states between Arabidopsis and rice (Fig. 3c and Fig. 4c). The distribution of in vivo RG4 folding scores in rice was shifted more to 1 compared to Arabidopsis (Fig. 3c and Fig. 4c). More RG4s in rice showed folding scores over 1 compared to the RG4s in Arabidopsis (Table S4 and S5). This result suggests that the folding landscape of RG4s is likely to be unique in different organisms under different growth conditions. Although previous chemical profiling in animal cell lines showed that RG4 structures are globally unfolded ${ }^{17}$, this might be due to the certain cell types having conditions not favourable for RG4 formation. Given the suggested functions of RG4s in special biological 
relevance, such as cancer cell growth and neurodegenerative diseases ${ }^{7,8,18,44,45}$, RG4 structures might stably form in specific cell types and growth conditions.

Previously, without direct evidence for RG4 formation in eukaryotes, it was not possible to infer whether suggested functions such as translation inhibition and splicing regulation are due to RG4 formation or specific sequence content ${ }^{8,9,46,47}$. In our study, we selected an in vivo folded RG4 located on HIRD11 to assess the functional impact of this RG4 structure (Fig. 5). The compelling phenotypic difference in root length between plants with and without RG4 structure indicated the significant impact of RG4 structure on plant development (Fig. 5). The direct evidence of in vivo RG4 formation substantiated by both genetic and biochemical validations provides the first demonstration of RG4 structure functionality in vivo. Given the large number of RG4s present in 340 plants (Figs. 3 and 4), further research is warranted to demonstrate that RG4s could significantly influence many aspects of plant growth and development. Apart from the translational regulation we revealed here (Fig.5), other biological functions such as splicing regulation might be also associated with RG4 structures ${ }^{47}$. Further exploration of the diverse functional roles of RG4 structures will expand our knowledge of RG4-dependent regulation of gene expression. 


\section{Acknowledgments}

We thank Prof. Giles Oldroyd (SLCU, Cambridge) and Dame Prof. Caroline Dean (John Innes

Centre), Sir Prof. Shankar Balasubramanian (U. Cambridge), Prof. Alison Smith (John Innes

Centre) and Dr. Desmond Bradley (John Innes Centre) for discussions with this work. This work

was supported by the Biotechnology and Biological Sciences Research Council (BBSRC:

BB/L025000/1 and BB/N022572/1) and the European Research Council (ERC: 680324) to Y.D..

M.I.U., J.Z., and C.K.K. were supported by Research Grants Council of the Hong Kong SAR

(CityU11100218, N_CityU110/17, CityU 21302317), Croucher Foundation (9500030, 9500039),

City University of Hong Kong (9680261), and the Petroleum Technology Development Fund

355 (Nigerian government). X.C. was supported by the National Natural Science Foundation of China (31788103/91540203) and the Chinese Academy of Sciences (QYZDY-SSW-SMC022).

\section{Author contributions}

X.Y. and Y.D. conceived the study; X.Y., C.K.K. and Y.D. designed the study; X.Y., Y.Z., H.D., 360 S.D., M.I.U., J.Z., Q.L., C.K.K. and Y.D. performed the experiments; X.Y. and J.C. did the analyses; X.C., C.K.K., and Y.D. supervised the analyses; X.Y., and Y.D. wrote the paper with input from all authors.

Competing interests: The authors declare no competing interest. 


\section{References}

1 Wan, Y., Kertesz, M., Spitale, R. C., Segal, E. \& Chang, H. Y. Understanding the transcriptome through RNA structure. Nat Rev Genet 12, 641-655, doi:10.1038/nrg3049 (2011).

2 Mortimer, S. A., Kidwell, M. A. \& Doudna, J. A. Insights into RNA structure and function

3 Rouskin, S., Zubradt, M., Washietl, S., Kellis, M. \& Weissman, J. S. Genome-wide probing of RNA structure reveals active unfolding of mRNA structures in vivo. Nature 505, 701705, doi:10.1038/nature12894 (2014).

4 Ganser, L. R., Kelly, M. L., Herschlag, D. \& Al-Hashimi, H. M. The roles of structural dynamics in the cellular functions of RNAs. Nat Rev Mol Cell Biol 20, 474-489, doi:10.1038/s41580-019-0136-0 (2019).

5 Fay, M. M., Lyons, S. M. \& Ivanov, P. RNA G-Quadruplexes in Biology: Principles and Molecular Mechanisms. J Mol Biol 429, 2127-2147, doi:10.1016/j.jmb.2017.05.017 (2017). doi:10.1016/j.tibtech.2017.06.012 (2017).

7 Haeusler, A. R. et al. C9orf72 nucleotide repeat structures initiate molecular cascades of disease. Nature 507, 195-200, doi:10.1038/nature13124 (2014).

Wolfe, A. L. et al. RNA G-quadruplexes cause eIF4A-dependent oncogene translation in cancer. Nature 513, 65-70, doi:10.1038/nature13485 (2014).

9 Cho, H. et al. Translational control of phloem development by RNA G-quadruplex-JULGI determines plant sink strength. Nat Plants 4, 376-390, doi:10.1038/s41477-018-0157-2 (2018).

10 Waldron, J. A., Raza, F. \& Le Quesne, J. eIF4A alleviates the translational repression mediated by classical secondary structures more than by G-quadruplexes. Nucleic Acids Res 46, 3075-3087, doi:10.1093/nar/gky108 (2018).

11 Biffi, G., Di Antonio, M., Tannahill, D. \& Balasubramanian, S. Visualization and selective chemical targeting of RNA G-quadruplex structures in the cytoplasm of human cells. Nat Chem 6, 75-80, doi:10.1038/nchem.1805 (2014).

12 Laguerre, A. et al. Visualization of RNA-Quadruplexes in Live Cells. J Am Chem Soc 137, 8521-8525, doi:10.1021/jacs.5b03413 (2015).

13 Yang, S. Y. et al. Transcriptome-wide identification of transient RNA G-quadruplexes in human cells. Nat Commun 9, 4730, doi:10.1038/s41467-018-07224-8 (2018).

14 Renard, I. et al. Small-molecule affinity capture of DNA/RNA quadruplexes and their identification in vitro and in vivo through the G4RP protocol. Nucleic Acids Res 47, 55025510, doi:10.1093/nar/gkz215 (2019).

15 Chen, S. B. et al. Visualization of NRAS RNA G-Quadruplex Structures in Cells with an Engineered Fluorogenic Hybridization Probe. J Am Chem Soc 138, 10382-10385, doi:10.1021/jacs.6b04799 (2016).

16 Chen, X. C. et al. Tracking the Dynamic Folding and Unfolding of RNA G-Quadruplexes in Live Cells. Angew Chem Int Ed Engl 57, 4702-4706, doi:10.1002/anie.201801999 (2018). 
17 Guo, J. U. \& Bartel, D. P. RNA G-quadruplexes are globally unfolded in eukaryotic cells and depleted in bacteria. Science 353, doi:10.1126/science.aaf5371 (2016).

18 Kharel, P., Balaratnam, S., Beals, N. \& Basu, S. The role of RNA G-quadruplexes in human diseases and therapeutic strategies. Wiley Interdiscip Rev RNA, e1568, doi:10.1002/wrna.1568 (2019).

19 Kwok, C. K., Sahakyan, A. B. \& Balasubramanian, S. Structural Analysis using SHALiPE to Reveal RNA G-Quadruplex Formation in Human Precursor MicroRNA. Angew Chem Int Ed Engl 55, 8958-8961, doi:10.1002/anie.201603562 (2016).

20 Wells, S. E., Hughes, J. M., Igel, A. H. \& Ares, M., Jr. Use of dimethyl sulfate to probe RNA structure in vivo. Methods Enzymol 318, 479-493, doi:10.1016/s00766879(00)18071-1 (2000).

21 Hagihara, M., Yoneda, K., Yabuuchi, H., Okuno, Y. \& Nakatani, K. A reverse transcriptase stop assay revealed diverse quadruplex formations in UTRs in mRNA. Bioorg Med Chem Lett 20, 2350-2353, doi:10.1016/j.bmcl.2010.01.158 (2010).

22 Britto, D. T. \& Kronzucker, H. J. Cellular mechanisms of potassium transport in plants. Physiol Plant 133, 637-650, doi:10.1111/j.1399-3054.2008.01067.x (2008).

23 Wang, M., Zheng, Q., Shen, Q. \& Guo, S. The critical role of potassium in plant stress response. Int J Mol Sci 14, 7370-7390, doi:10.3390/ijms14047370 (2013).

24 Kwok, C. K., Ding, Y., Shahid, S., Assmann, S. M. \& Bevilacqua, P. C. A stable RNA Gquadruplex within the 5'-UTR of Arabidopsis thaliana ATR mRNA inhibits translation. Biochem J 467, 91-102, doi:10.1042/BJ20141063 (2015).

25 Kwok, C. K., Marsico, G., Sahakyan, A. B., Chambers, V. S. \& Balasubramanian, S. rG4seq reveals widespread formation of G-quadruplex structures in the human transcriptome. Nat Methods 13, 841-844, doi:10.1038/nmeth.3965 (2016).

26 Bugaut, A., Rodriguez, R., Kumari, S., Hsu, S. T. \& Balasubramanian, S. Small moleculemediated inhibition of translation by targeting a native RNA G-quadruplex. Org Biomol Chem 8, 2771-2776, doi:10.1039/c002418j (2010).

27 Mullen, M. A. et al. RNA G-Quadruplexes in the model plant species Arabidopsis thaliana: prevalence and possible functional roles. Nucleic Acids Res 38, 8149-8163, doi:10.1093/nar/gkq804 (2010).

28 Lorenz, R. et al. ViennaRNA Package 2.0. Algorithms Mol Biol 6, 26, doi:10.1186/17487188-6-26 (2011).

29 Kwok, C. K., Ding, Y., Tang, Y., Assmann, S. M. \& Bevilacqua, P. C. Determination of in vivo RNA structure in low-abundance transcripts. Nat Commun 4, 2971, doi:10.1038/ncomms3971 (2013).

30 Ding, Y. et al. In vivo genome-wide profiling of RNA secondary structure reveals novel regulatory features. Nature 505, 696-700, doi:10.1038/nature12756 (2014).

31 Wang, Z., Wang, M., Wang, T., Zhang, Y. \& Zhang, X. Genome-wide probing RNA structure with the modified DMS-MaPseq in Arabidopsis. Methods 155, 30-40, doi:10.1016/j.ymeth.2018.11.018 (2019).

32 Spitale, R. C. et al. RNA SHAPE analysis in living cells. Nat Chem Biol 9, 18-20, doi:10.1038/nchembio.1131 (2013).

33 Seck, P. A., Diagne, A. \& Wopereis, M. C. S. Crops that feed the world 7: Rice. Food Security 4, 7-24, doi:https://doi.org/10.1007/s12571-012-0168-1 (2012). 
34 Missra, A. \& von Arnim, A. G. Analysis of mRNA translation states in Arabidopsis over the diurnal cycle by polysome microarray. Methods Mol Biol 1158, 157-174,

455 doi:10.1007/978-1-4939-0700-7_10 (2014).

$35 \mathrm{Li}, \mathrm{W}$. et al. EIN2-directed translational regulation of ethylene signaling in Arabidopsis. Cell 163, 670-683, doi:10.1016/j.cell.2015.09.037 (2015).

36 Rhodes, D. \& Lipps, H. J. G-quadruplexes and their regulatory roles in biology. Nucleic Acids Res 43, 8627-8637, doi:10.1093/nar/gkv862 (2015).

37 Dhapola, P. \& Chowdhury, S. QuadBase2: web server for multiplexed guanine quadruplex mining and visualization. Nucleic Acids Res 44, W277-283, doi:10.1093/nar/gkw425 (2016).

38 Saad, M. et al. Mapping and characterization of G-quadruplexes in the genome of the social amoeba Dictyostelium discoideum. Nucleic Acids Res 47, 4363-4374, doi:10.1093/nar/gkz196 (2019).

39 Endoh, T. \& Sugimoto, N. Conformational Dynamics of the RNA G-Quadruplex and its Effect on Translation Efficiency. Molecules 24, doi:10.3390/molecules24081613 (2019).

40 Lightfoot, H. L., Hagen, T., Tatum, N. J. \& Hall, J. The diverse structural landscape of quadruplexes. FEBS Lett 593, 2083-2102, doi:10.1002/1873-3468.13547 (2019).

41 Pandey, S., Agarwala, P. \& Maiti, S. Effect of loops and G-quartets on the stability of RNA G-quadruplexes. J Phys Chem B 117, 6896-6905, doi:10.1021/jp401739m (2013).

42 Binzel, M. L., Hess, F. D., Bressan, R. A. \& Hasegawa, P. M. Intracellular compartmentation of ions in salt adapted tobacco cells. Plant Physiol 86, 607-614, doi:10.1104/pp.86.2.607 (1988).

43 Dreyer, I. \& Uozumi, N. Potassium channels in plant cells. FEBS J 278, 4293-4303, doi:10.1111/j.1742-4658.2011.08371.x (2011).

44 Fay, M. M., Anderson, P. J. \& Ivanov, P. ALS/FTD-Associated C9ORF72 Repeat RNA Promotes Phase Transitions In Vitro and in Cells. Cell Rep 21, 3573-3584, doi:10.1016/j.celrep.2017.11.093 (2017). disease. Nat Rev Neurol 14, 544-558, doi:10.1038/s41582-018-0047-2 (2018).

Arora, A. et al. Inhibition of translation in living eukaryotic cells by an RNA G-quadruplex motif. RNA 14, 1290-1296, doi:10.1261/rna.1001708 (2008).

Huang, H., Zhang, J., Harvey, S. E., Hu, X. \& Cheng, C. RNA G-quadruplex secondary structure promotes alternative splicing via the RNA-binding protein hnRNPF. Genes Dev 31, 2296-2309, doi:10.1101/gad.305862.117 (2017).

48 O'Malley R.C., B. C. C., Ecker J.R. in Plant Functional Genomics. Methods in Molecular Biology Vol. 1284 (ed Stepanova A. Alonso J.) (Humana Press, 2015).

49 Zhang, X., Henriques, R., Lin, S. S., Niu, Q. W. \& Chua, N. H. Agrobacterium-mediated transformation of Arabidopsis thaliana using the floral dip method. Nat Protoc 1, 641-646, doi:10.1038/nprot.2006.97 (2006).

50 Heckman, K. L. \& Pease, L. R. Gene splicing and mutagenesis by PCR-driven overlap extension. Nat Protoc 2, 924-932, doi:10.1038/nprot.2007.132 (2007).

51 Ding, Y., Kwok, C. K., Tang, Y., Bevilacqua, P. C. \& Assmann, S. M. Genome-wide profiling of in vivo RNA structure at single-nucleotide resolution using structure-seq. Nat Protoc 10, 1050-1066, doi:10.1038/nprot.2015.064 (2015).

52 Langmead, B. Aligning short sequencing reads with Bowtie. Curr Protoc Bioinformatics Chapter 11, Unit 11 17, doi:10.1002/0471250953.bi1107s32 (2010). 
53 Norris, M. et al. FoldAtlas: a repository for genome-wide RNA structure probing data. Bioinformatics 33, 306-308, doi:10.1093/bioinformatics/btw611 (2017).

54 Li, H. et al. The Sequence Alignment/Map format and SAMtools. Bioinformatics 25, 2078 2079, doi:10.1093/bioinformatics/btp352 (2009). 


\section{Figure legends}

Fig.1 rG4-seq reveals the global landscape of G-rich regions with the potential to fold into RG4 structures in Arabidopsis.

a Schematic of an RNA G-quadruplex (RG4). The schematic depicts an RG4 structure with three layers of G-quartets (G3 RG4, guanine (G) coloured in orange), with the loop length of any nucleotide $\left(\mathrm{N}\right.$, coloured in grey), potassium ions $\left(\mathrm{K}^{+}\right.$, grey ball) coordinated within the G-quartets stabilize RG4s.

b Workflow of rG4-seq. Poly(A) enriched RNAs were subjected to reverse transcription under the buffers with $\mathrm{Li}^{+}$(non-stabilizing condition), $\mathrm{K}^{+}$(stabilizing condition) or $\mathrm{K}^{+}+\mathrm{PDS}$ (stronger stabilizing condition), respectively. The G-rich region sites with folding potential were identified by comparing the coverage of reads between the rG4-seq libraries with different cations as described above.

c rG4-seq profiles of $A t S M X L 5$ displayed the reads coverage of reverse transcription (RT) with $\mathrm{Li}^{+}$ (top), $\mathrm{K}^{+}$(middle), and $\mathrm{K}^{+}+\mathrm{PDS}$ (bottom) respectively. The 3 'end of the G-rich region is indicated by red triangle. A (blue), C (light grey), G(yellow), U(green).

d Residue distribution around RTS sites. Guanine $(\mathrm{G})$ was strongly enriched in the upstream sequences of RT stalling (RTS) identified under both $\mathrm{K}^{+}$and $\mathrm{K}^{+}+\mathrm{PDS}$ conditions, but not in the transcriptome and the downstream sequences of RTS. A (blue), C (light grey), G(yellow), $530 \quad \mathrm{U}($ green $)$.

e Classification of G-rich regions with folding potential. G-rich regions with folding potential identified in $\mathrm{K}^{+}$(dark blue) and $\mathrm{K}^{+}+\mathrm{PDS}$ conditions (black) were classified into six categories according to the number of G-quartets (G2 with two G-quartets or G3 with three G-quartets), loop length (L, 1-15 nt), and bulge size (non-canonical G3 RG4s with a guanine vacancy: G3VL1-9, or a bulge: G3bulge).

f The prevalence of G-rich regions in different genic regions.

g and $\mathbf{h}$ rG4-seq profiles of G2 G-rich region on AT4G30460 (g) and G3 G-rich region on $A T 3 G 23450(\mathrm{~h})$, otherwise in Fig. 1c.

i Comparison of base-pairing probability (BPP) of alternative secondary structure in G-rich regions that are detected with $\mathrm{K}^{+}$(blue) and undetected (grey) using rG4-seq. The Gs in the G-rich region were classed to 8 bins, flanking sequences (100 nt on both sides) were classed to 20 bins, with 15 bins close to G-rich regions shown. Differences of BPPs between G-rich regions and flanking regions, detected by $\mathrm{rG} 4$ with $\mathrm{K}^{+}, P=0.444$; undetected regions, $P<10^{-16}$; $P$-values, paired Student's t-test.

j Secondary structure of G-rich region detected by rG4-seq (in Fig. 1h) and flanking sequences on AT3G23450, predicted using Vienna RNAfold. The filling colours of orange, green and blue indicate the base-pairing probability of below $0.3,0.3-0.7$ and above 0.7 respectively. Red stars 
indicate the guanines comprising the G3 region. Numbers indicate nucleotide positions on the transcript.

\section{Fig. 2 SHALiPE-Seq determines folding states of G-rich regions robustly.}

a Schematic of SHALiPE-Seq in vitro with $\mathrm{K}^{+}$and $\mathrm{Li}^{+}$. In vitro probing in the presence of $\mathrm{K}^{+}$and $560 \mathrm{Li}^{+}$established the benchmarks of folded and unfolded states of G-rich regions respectively. NAI (indicated by red cross) preferentially modifies the last $\mathrm{G}$ in $\mathrm{G}$ tracts of folded RG4s, resulting in a high Gini index of read counts (reads on preferentially modified Gs were in red) in SHALiPE profiles. In contrast, the distribution of the SHALiPE profile for the unfolded state in the presence of $\mathrm{Li}^{+}$is uniform, resulting in a low Gini.

b For G-rich regions detected using rG4-seq in the presence of $\mathrm{K}^{+}$, Gini of SHALiPE profiles in vitro with $\mathrm{K}^{+}$(folded state) was greater than that of $i$ vitro with $\mathrm{Li}^{+}$(unfolded state) by a factor of 1.20 ( $\mathrm{n}=117, P$-value, paired Student's t-test, average reads coverage on $\mathrm{G} \geq 50$ reads/nt).

c and d rG4-seq profiles of G2 G-rich region on AT5G05380 (c) and G3 G-rich region on $A T 5 G 62670$ (d). The 3 'end of the G-rich region is indicated by a red triangle. A (blue), C (light grey), G(yellow), U(green).

e and $\mathbf{f}$ SHALiPE profiles in vitro with $\mathrm{K}^{+}$or $\mathrm{Li}^{+}$of $\mathrm{G} 2 \mathrm{G}$-rich region on $A T 5 G 05380$ (c) and G3 G-rich region on $A T 5 G 62670$ (d). High read counts of last guanines of G-tracts (indicated by black arrows) represent strong modifications of NAI on these guanines in the presence of $\mathrm{K}^{+}$, indicating $\mathrm{RG} 4 \mathrm{~s}$ are folded. In the presence of $\mathrm{Li}^{+}$, last $\mathrm{Gs}$ are not strongly modified, representing unfolded state of these G-rich regions. The Gini values with $\mathrm{K}^{+}$are higher than those with $\mathrm{Li}^{+}$, as indicated. A (blue), C (light grey), G(yellow), U(green).

Fig. 3 In vivo SHALiPE-Seq reveals hundreds of folded RG4s in Arabidopsis.

a Schematic of in vivo SHALiPE-Seq in Arabidopsis. In vivo folding state of G-rich region is evaluated by comparing SHALiPE profiles in vivo with SHALiPE profiles in vitro in the presence of $\mathrm{Li}^{+}$or $\mathrm{K}^{+}$respectively.

b Cumulative plot of Gini index of Arabidopsis G-rich regions in vivo, in vitro with $\mathrm{Li}^{+}$and in vitro with $\mathrm{K}^{+}$. A significantly higher Gini in vivo than that in vitro with $\mathrm{Li}^{+}$indicates the folding state of G-rich regions in vivo. $P$-value, paired Student's t-test.

c Histogram of in vivo folding score (FS) in Arabidopsis. The median value is 0.755 . The FS of 0 represents the unfolded states (with $\mathrm{Li}^{+}$) and 1 represents the folded states of $\mathrm{RG} 4 \mathrm{~s}$ (with $\mathrm{K}^{+}$) in vitro.

d and e SHALiPE profiles of the RG4 region on AT4G30460 (d) and AT3G2345(e). The in vivo SHALiPE profile resembled the in vitro SHALiPE profile with $\mathrm{K}^{+}$(the last Gs of G-tracts indicated 
600

605

by black arrows) but not the in vitro SHALiPE profile with $\mathrm{Li}^{+}$, indicating the folded state of this RG4 in vivo.

f Violin plot of in vivo folding scores of G-rich regions of different types. Folding scores of RG4s of different catalogues are similar ( $P$ values $>0.05$, one-way ANOVA/ Tukey HSD post hoc test).

g Violin plot of in vivo folding scores of G-rich regions in CDS and UTRs. Folding scores of RG4s of different genic regions are similar ( $P>0.05$, one-way ANOVA/ Tukey HSD post hoc test).

\section{Fig. 4 In vivo SHALiPE-Seq reveals hundreds of folded RG4s in rice.}

a Schematic of in vivo SHALiPE-Seq in rice. The in vivo folding state of G-rich region is evaluated by comparing SHALiPE profiles in vivo with SHALiPE profiles in vitro in the presence of $\mathrm{Li}^{+}$or $\mathrm{K}^{+}$respectively.

b Cumulative plot of Gini index of rice G-rich regions in vivo, in vitro with $\mathrm{Li}^{+}$and in vitro with $\mathrm{K}^{+}$. A significantly higher Gini in vivo than that in vitro with $\mathrm{Li}^{+}$indicates the folding state of Grich regions in vivo. $P$-value, paired Student's t-test.

c Histogram of in vivo folding score (FS) in rice. The median value is 0.938 . The FS of 0 represents the unfolded states (with $\mathrm{Li}^{+}$) and 1 represents the folded states of $\mathrm{RG} 4 \mathrm{~s}$ (with $\mathrm{K}^{+}$) in vitro.

d and e SHALiPE profiles of the RG4 region on LOC_Os02g15810(d) and LOC_Os07g41694(e). The in vivo SHALiPE profile resembled the in vitro $\mathrm{SHALiPE}$ profile with $\mathrm{K}^{+}$(the last Gs of Gtracts indicated by black arrows) but not the in vitro SHALiPE profile with $\mathrm{Li}^{+}$, indicating the folded state of this RG4 in vivo.

f Violin plot of in vivo folding scores of G-rich regions of different types. Folding scores of RG4s of different catalogues are similar ( $P$ values $>0.05$, one-way ANOVA/ Tukey HSD post hoc test).

g Violin plot of in vivo folding scores of G-rich regions in CDS and UTRs. Folding scores of RG4s of different genic regions are similar ( $P>0.05$, one-way ANOVA/ Tukey HSD post hoc test).

\section{Fig. 5 RG4 regulates plant growth and translation.}

a SHALiPE profiles of the RG4 region on 3'UTR of HIRD11 (AT1G54410) for in vivo, in vitro with $\mathrm{K}^{+}$and in vitro with $\mathrm{Li}^{+}$. The in vivo SHALiPE profile resembled the in vitro SHALiPE profile with $\mathrm{K}^{+}$(the last $\mathrm{G}$ in $\mathrm{G}$ tracts of the $\mathrm{RG} 4$ region indicated by black arrows) but not the in vitro SHALiPE profile with $\mathrm{Li}^{+}$, indicating the folded state of this RG4 in vivo.

b Schematic diagram of HIRD11 showing the T-DNA insertion site of hird11-1 (GABI_494_A09).

c Relative mRNA abundance of HIRD11 in Col-0 and hird11-1 plants indicated the hird11-1 is a knock-down mutant, error bars indicate SE. 
645 d Sequences of wildtype RG4 (wtRG4, left) and disrupted G-rich region with G to A mutation (mutRG4, right) on HIRD11.

e and f RG4 modulates plant growth. Representative 6-day-old plants of Col-0, hird11-1, complemented hird11-1 with wildtype RG4 (hird11-1-comp-wtRG4) and complemented hird11-1 650 with mutated RG4 (hird11-1-comp-mutRG4) (e); and average primary root lengths (f) of more than 20 plants for each genotype. Significant differences were evaluated by one-way ANOVA/ Tukey HSD post hoc test $(P<0.05)$. Error bars indicate SE.

655 HIRDI1 in each polysome associated fraction was detected by qRT-PCR and quantified as a percentage relative to their total amount, error bars indicate SE. 


\section{Figure 1}

a

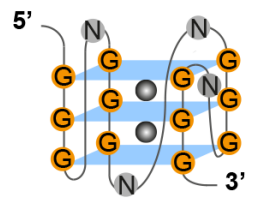

b

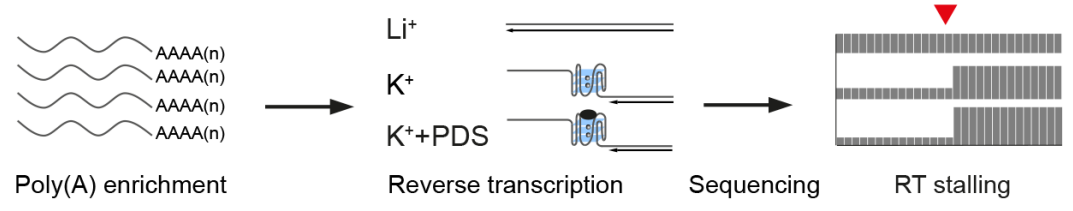

C

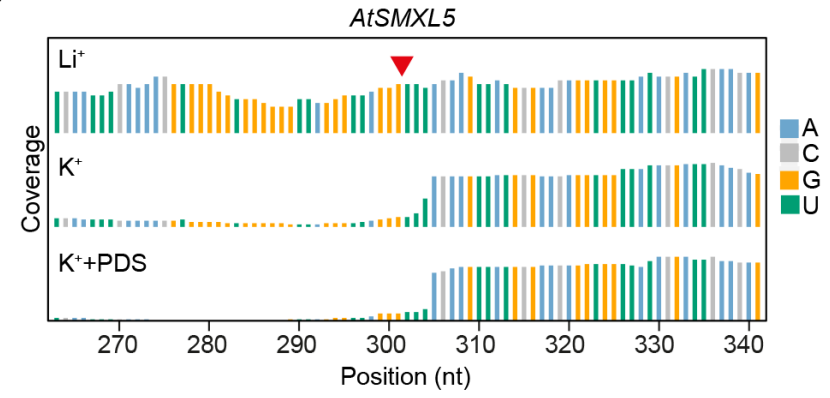

e

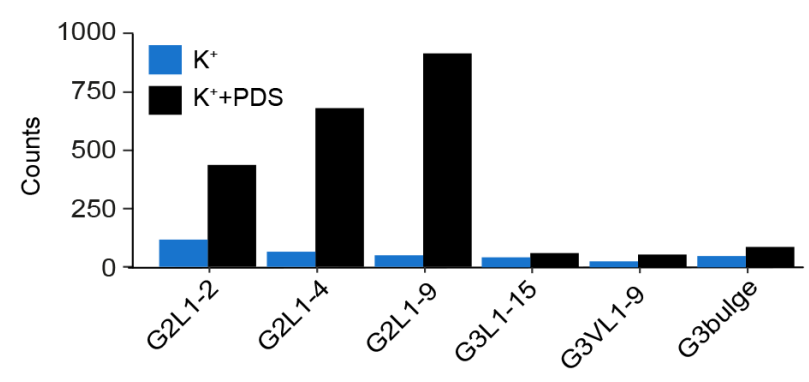

d

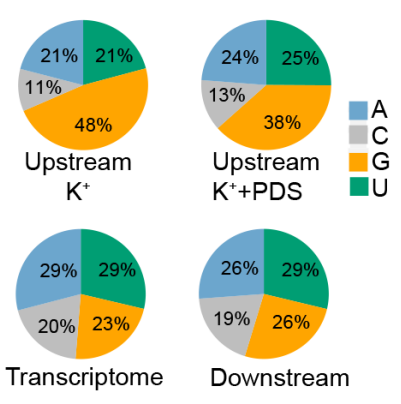

f

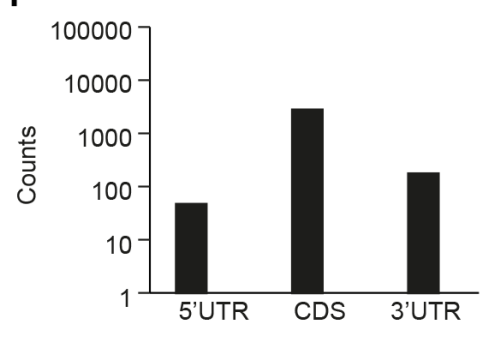

g

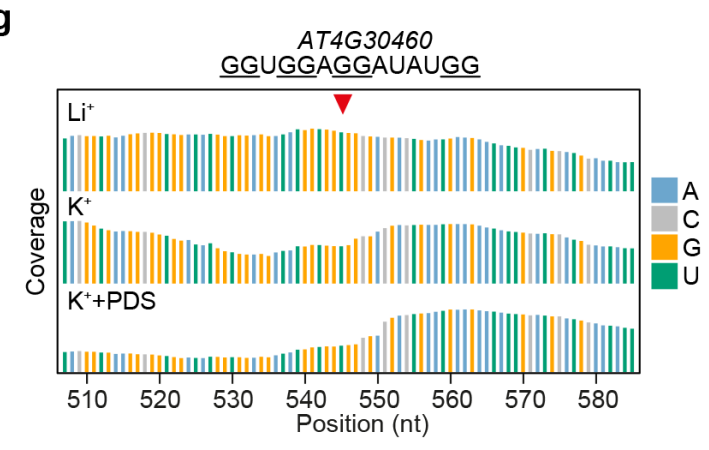

i

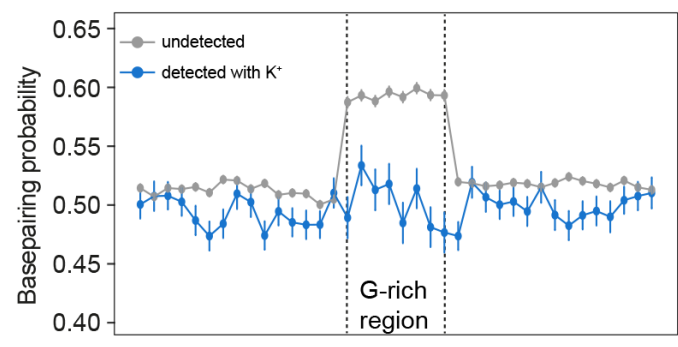

h

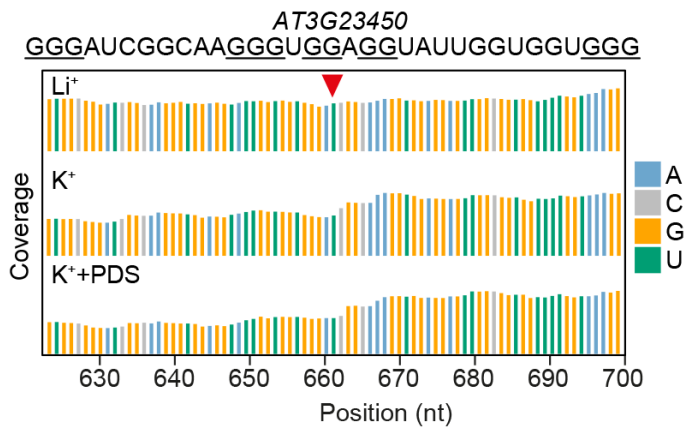

j

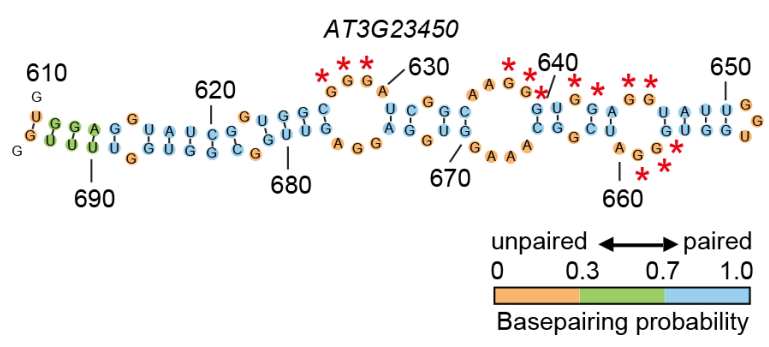


Figure 2

a

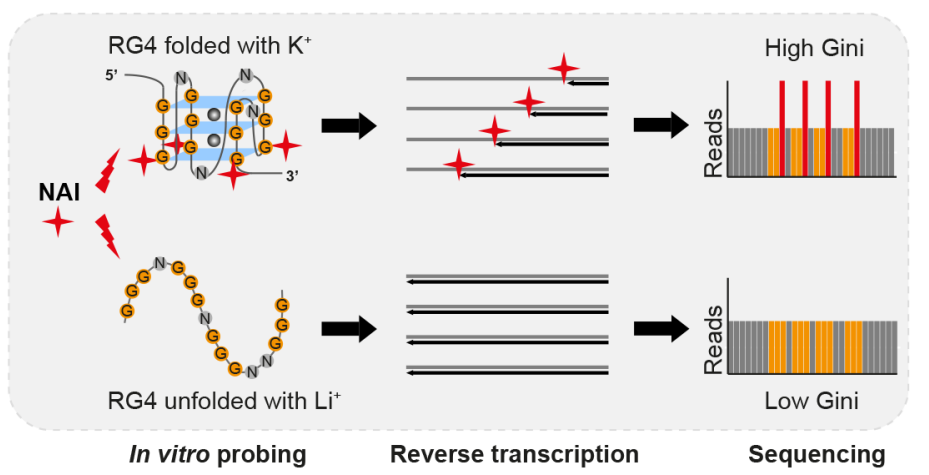

b

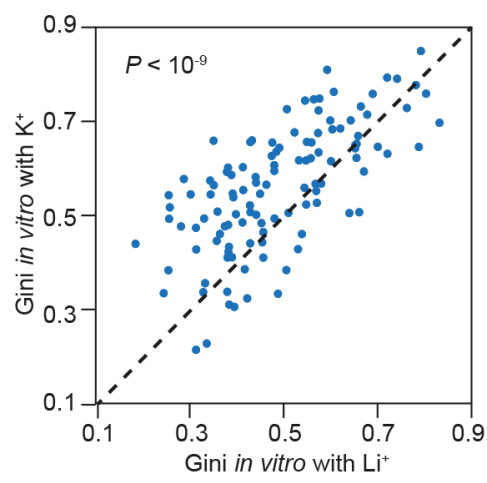

C

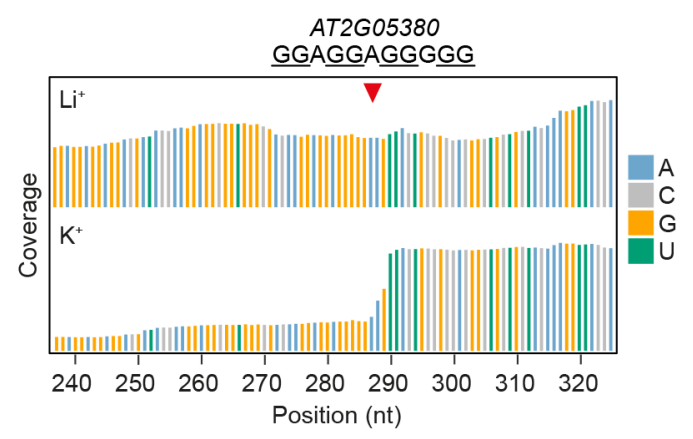

e
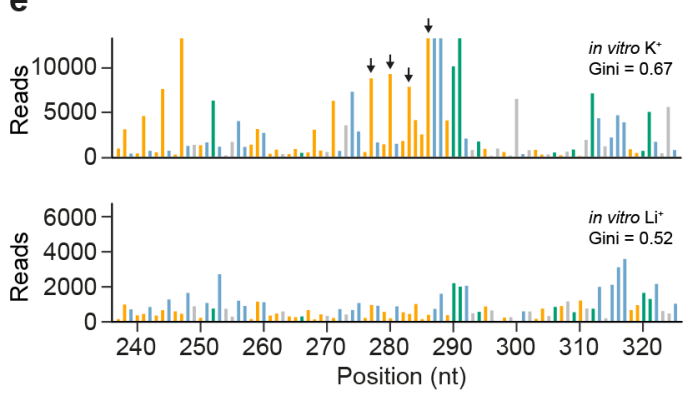

d AT5G62670 GGGUGGGGAUGGGCCGGGG

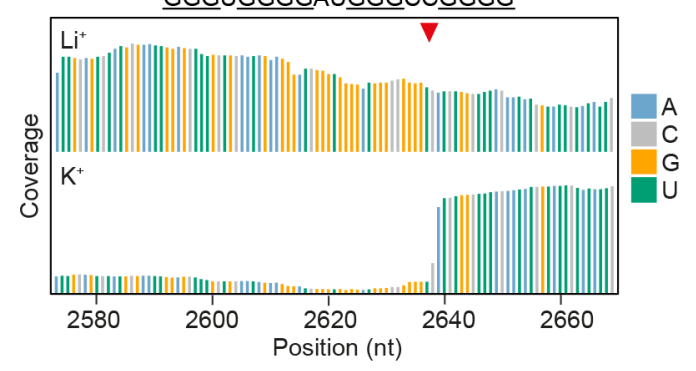

f

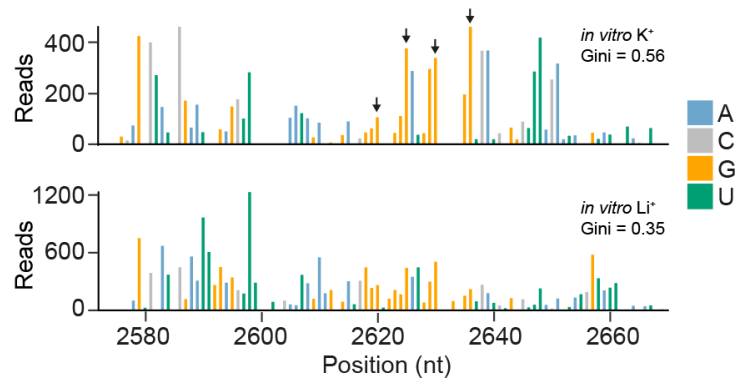


Figure 3

a

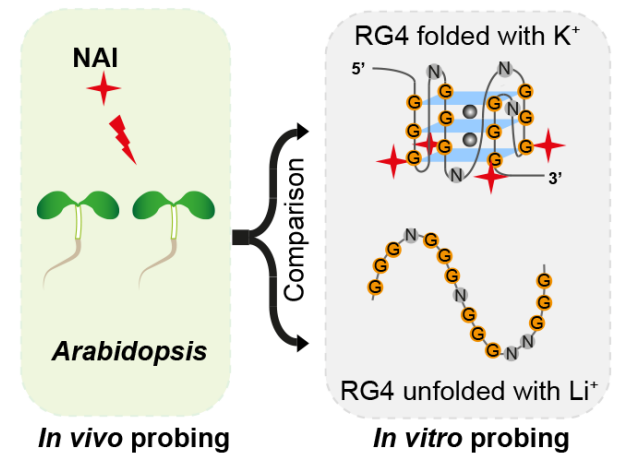

b

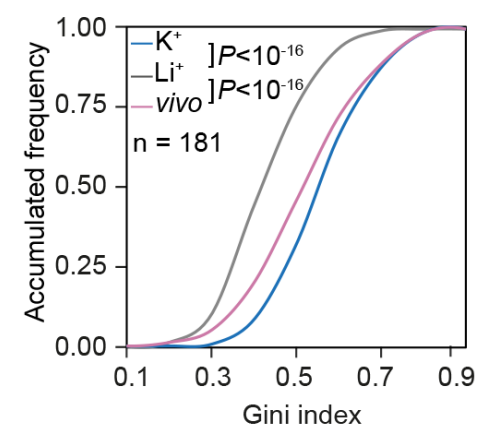

C

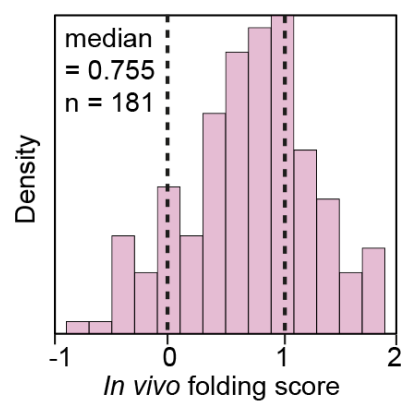

d

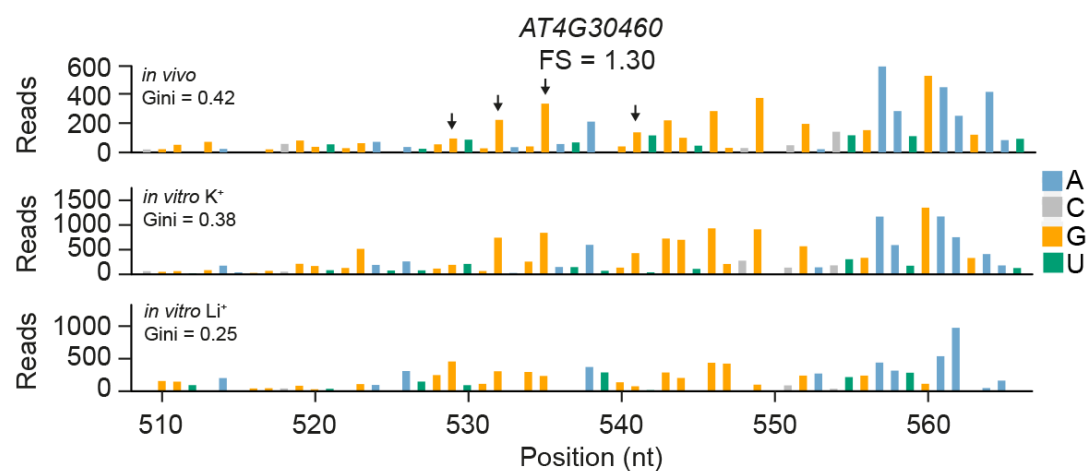

e

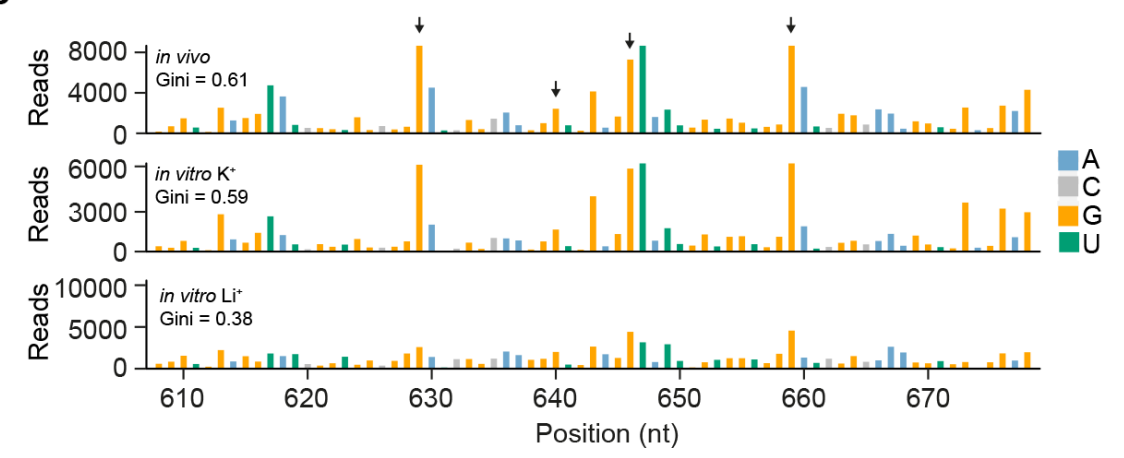

f

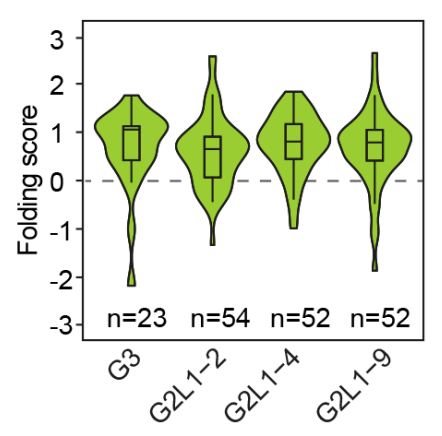

$\mathbf{g}$

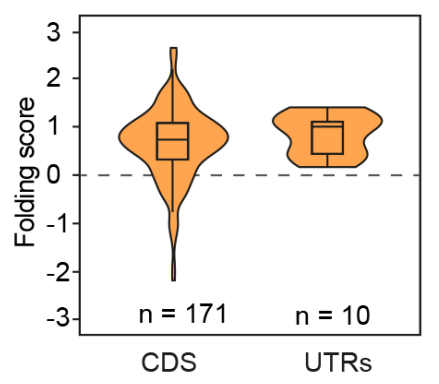




\section{Figure 4}

a

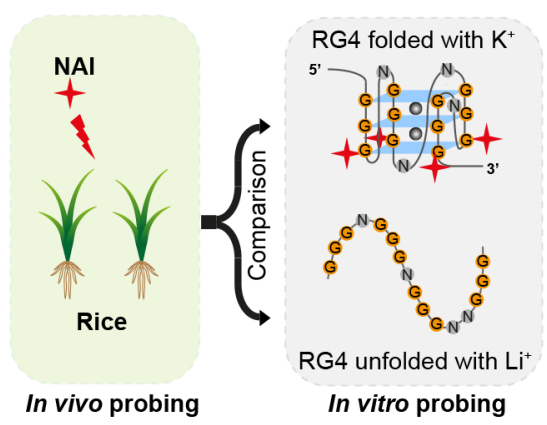

d

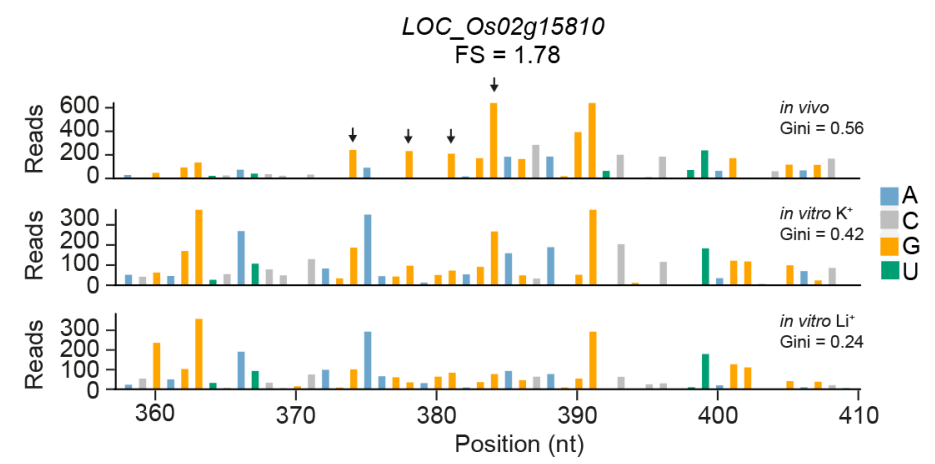

e

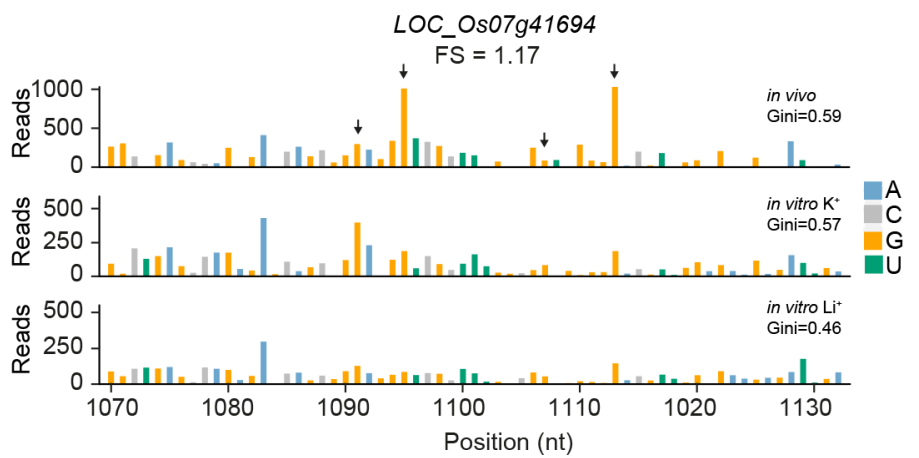

C
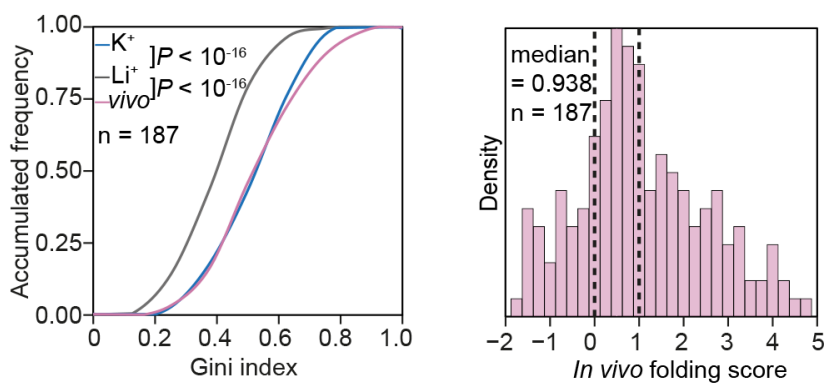

f

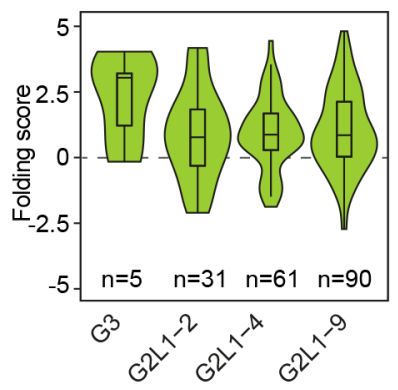

g

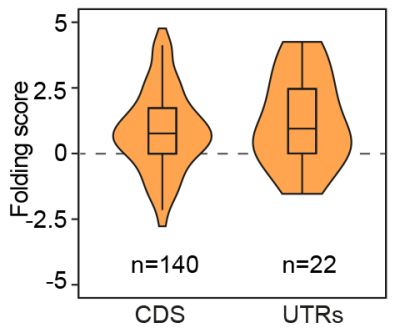


Figure 5

a

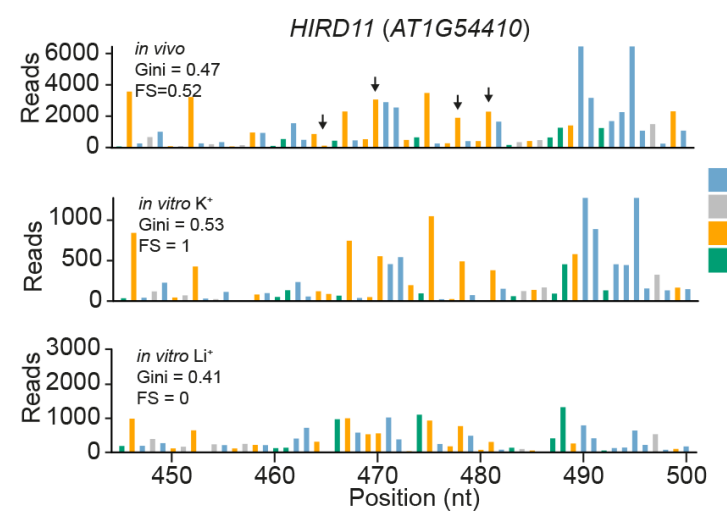

e

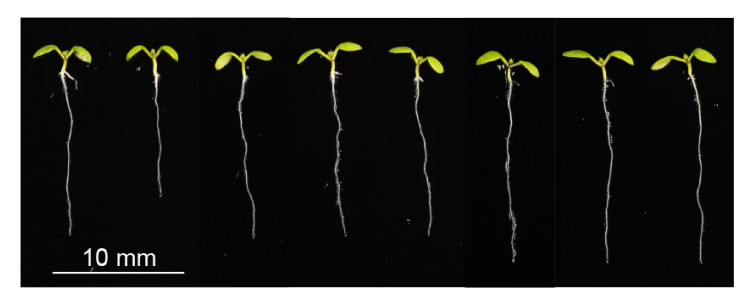

Col-0 hird11-1 $\begin{array}{rrrrr}\# 4 & \# 6 \quad \# 7 & \# 6 & \# 10 \quad \# 18 \\ \text { hird11-1-comp-wtRG4 } & \text { hird11-1-comp-mutRG } 4\end{array}$ b

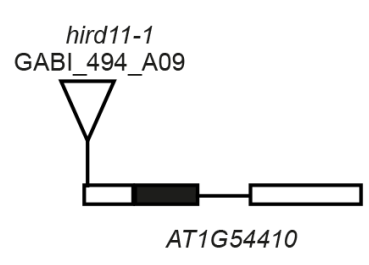

d GGTGAGGAAGTGAGGAGG

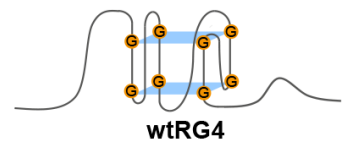

f

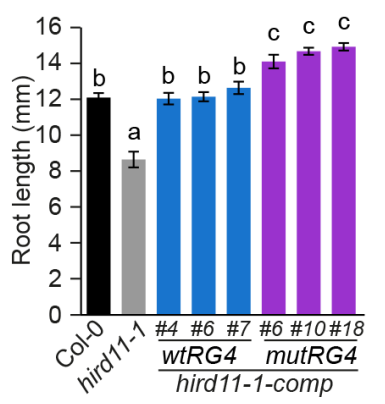

C

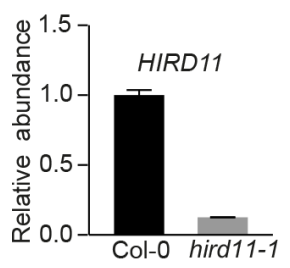

GGTGAaaAGTGAaaAGG

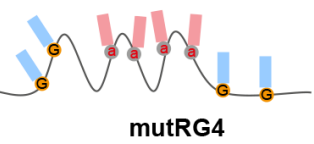

g

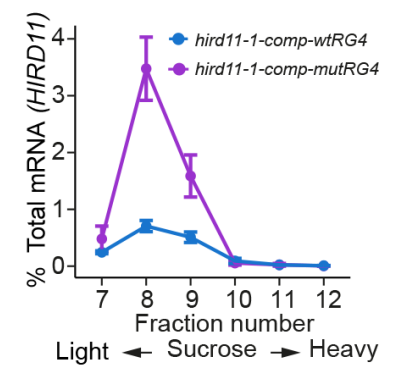

